\title{
Mathematics education with/for indigenous peoples
}

\author{
Robyn Jorgensen • David Wagner
}

Published online: 8 February 2013

(C) Mathematics Education Research Group of Australasia, Inc. 2013

Within mathematics education, research in indigenous contexts has changed dramatically over the past few decades with the emphasis shifting from researching on indigenous people to researching with indigenous people. Along with the changes in the way research is done in these contexts, there is significant growth in the number of research projects. This growth can be seen, for example, in the numbers of papers in this area in the conference proceedings of the Mathematics Education Research Group of Australasia (MERGA). This development was an important impetus for this Special Issue of the Mathematics Education Research Journal (MERJ) focused on indigenous people and their access to school mathematics.

While there are diverse approaches and ideologies that underpin research projects in indigenous contexts, there are some common denominators that guide research. This Special Issue represents a collection of research projects in which the intent has been to work with indigenous people to enhance the mathematics learning for indigenous students. In our call for papers, we sought contributions with the following characteristics:

- the research is with and/or by, rather than on, indigenous people,

- authors do not approach their work through the exoticising of indigenous people,

- the research projects attempt to push learning rather than rely on deficit models, and

- the research pushes boundaries and challenges some of the existing orthodoxies that perpetuate differences in learning and outcomes.

Our collaboration on this project grew from a long-standing involvement of the two editors in this area. Drawing on our work in the Southern and Northern

\footnotetext{
R. Jorgensen $(\bowtie)$

Griffith University, South East Queensland, Australia

e-mail: r.jorgensen@griffith.edu.au

D. Wagner

University of New Brunswick, New Brunswick, Canada

e-mail: dwagner@unb.ca
} 
hemispheres, we wanted this issue to be a collection of international research. Our interest and work in indigenous education enabled us to link in with many networks and researchers who work in this area, and this collection of work represents a significant breadth of work that is being undertaken across the globe. We were keen to include authors from all continents but were unable to achieve this goal. However, we are very pleased with the contributions contained within this Issue. We acknowledge the contribution of the authors as well as the many communities and people with whom they work.

In circulating among our many communities, we were keen to ensure that this issue captured the international work that is being undertaken in collaboration with indigenous people and/or schools/communities. We wanted to ensure that the papers for this Special Issue were clearly different from those of the past where there was an imposition on indigenous people to be research subjects, which runs the risk of exotising of the "Other."

The range of research relationships represented in the articles of this issue highlight several realities for research in indigenous contexts. We would argue that these realities are present in all research relationships, but the interaction of cultures in indigenous contexts helps us to see the realities more clearly. First, we note that there are various people within indigenous communities with whom a researcher might relate. We researchers can interact with indigenous children in schools, indigenous scholars, indigenous teachers, and indigenous elders or leaders in a community. We might work with and support others who interact with these people too. Through this diversity of relationships, the qualities of the interaction are of utmost importance. How do we listen? Whose worldviews or storylines are used to interpret the voices of the indigenous people in our relationships?

A more complex question relates to the invitation to engage with a community. Clearly, such an invitation implies an expectation that we bring into the community some of our knowledge and sensibility (which are bound to cultures outside the community); this is why the community invites an outsider in. The difference between local culture and the invited-in outside culture is especially strong in the context of mathematics learning, because of a widespread (but false) view that mathematical knowledge is independent of context. Yet we know that an invitation into a community also demands an expectation to be responsive to the knowledge and sensibility of the community. This tension is part of any engagement we have with the people in an indigenous community. This would be true even of an indigenous scholar bringing a scholarly orientation into an indigenous community, but the tension is greater for non-indigenous scholars invited into a community.

While editing this Special Issue, we noticed that this cross-cultural reality is especially significant in regard to scholarly review. The traditions of academic scholarship have people outside the community relationship judging the qualities of interaction among the researchers and the community members inside the community. When we review reports on research in indigenous communities we look for articulation of the research relationship and expect certain signs of respectful interaction. The problem is that we rely on our own experiences of interacting with people in different indigenous communities to judge the relationship in a community we never experienced. This is a form of generalisation that violates the honouring of traditions and expectations in the community in which the research took place. This 
problem exists for any reader of the articles in this Special Issue (or of any reports on research in indigenous contexts), but the judgment of a reader is less influential than the judgment of a reviewer. Though this problem of judgment from outside the community is real, the alternative is worse. We would not want to abandon the opportunity to learn from the stories of other scholars' engagement with particular communities.

In the screening of articles for this Special Issue we have been careful to require description of respectful relationships in the communities researched, but at the same time to allow for diverse relationships that are responsive to the people in the communities. The authors in this issue have worked alongside indigenous people to support leading and learning in school mathematics. The authors are from very diverse contexts and worked on very different approaches to address a significant issue. This collection is a testament to the many projects, and many foci, that are integral to creating opportunities for access and success.

As the authors in this issue share their work in the MERJ arena, it becomes very clear to readers that there is no one single approach, no single theme that can be used to address the breadth of challenges. The authors highlight the significant work that is being undertaken internationally.

All papers in this issue have gone through the usual MERJ review process but we have been very keen to ensure that local work has been handled by the editor who is not familiar with that context. This is the first Special Issue of MERJ since we have used the fully on-line process with Springer so it has been a learning process for the Editorial team, the Editorial Board members, the Special Issue Editors, and our authors. For this reason, the Editor-in-Chief was one of the Editors so that we could manage the new system and processes which will enable future Special Issues to work well. We would like to thank our reviewers, authors, Editorial Board members, and the Springer team for helping ensure a successful first on-line issue of the MERJ Special Issues. 